\title{
Article \\ Stability and Reproducibility of Radiomic Features Based Various Segmentation Technique on MR Images of Hepatocellular Carcinoma (HCC)
}

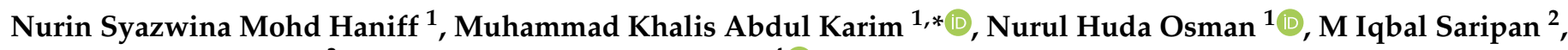 \\ Iza Nurzawani Che Isa ${ }^{3}$ and Mohammad Johari Ibahim ${ }^{4}$ (D) \\ 1 Department of Physics, Faculty of Science, Universiti Putra Malaysia, Serdang 43400, Selangor, Malaysia; \\ nurinsyazwina1996@gmail.com (N.S.M.H.); nurulhuda@upm.edu.my (N.H.O.) \\ 2 Department of Computer Engineering, Faculty of Engineering, Universiti Putra Malaysia, \\ Serdang 43400, Selangor, Malaysia; iqbal@upm.edu.my \\ 3 Programme of Diagnostic Imaging and Radiotherapy, Universiti Kebangsaan Malaysia, \\ Kuala Lumpur 50300, Wilayah Persekutuan, Malaysia; zawani@ukm.edu.my \\ 4 Department Biochemistry \& Molecular Medicine, Faculty of Medicine, Universiti Teknologi MARA, \\ Sungai Buloh 47200, Selangor, Malaysia; mji@uitm.edu.my \\ * Correspondence: mkhalis@upm.edu.my
}

Citation: Haniff, N.S.M.; Abdul Karim, M.K.; Osman, N.H.; Saripan, M.I.; Che Isa, I.N.; Ibahim, M.J. Stability and Reproducibility of Radiomic Features Based Various Segmentation Technique on MR Images of Hepatocellular Carcinoma (HCC). Diagnostics 2021, 11, 1573. https://doi.org/10.3390/

diagnostics11091573

Academic Editors: Saif Afat and Thomas Geyer

Received: 9 July 2021

Accepted: 23 August 2021

Published: 30 August 2021

Publisher's Note: MDPI stays neutral with regard to jurisdictional claims in published maps and institutional affiliations.

Copyright: (c) 2021 by the authors. Licensee MDPI, Basel, Switzerland. This article is an open access article distributed under the terms and conditions of the Creative Commons Attribution (CC BY) license (https:// creativecommons.org/licenses/by/ $4.0 /)$.

\begin{abstract}
Hepatocellular carcinoma (HCC) is considered as a complex liver disease and ranked as the eighth-highest mortality rate with a prevalence of $2.4 \%$ in Malaysia. Magnetic resonance imaging (MRI) has been acknowledged for its advantages, a gold technique for diagnosing HCC, and yet the false-negative diagnosis from the examinations is inevitable. In this study, $30 \mathrm{MR}$ images from patients diagnosed with HCC is used to evaluate the robustness of semi-automatic segmentation using the flood fill algorithm for quantitative features extraction. The relevant features were extracted from the segmented MR images of HCC. Four types of features extraction were used for this study, which are tumour intensity, shape feature, textural feature and wavelet feature. A total of 662 radiomic features were extracted from manual and semi-automatic segmentation and compared using intraclass relation coefficient (ICC). Radiomic features extracted using semi-automatic segmentation utilized flood filling algorithm from 3D-slicer had significantly higher reproducibility (average ICC $=0.952 \pm 0.009, p<0.05$ ) compared with features extracted from manual segmentation (average $\mathrm{ICC}=0.897 \pm 0.011, p>0.05$ ). Moreover, features extracted from semi-automatic segmentation were more robust compared to manual segmentation. This study shows that semi-automatic segmentation from 3D-Slicer is a better alternative to the manual segmentation, as they can produce more robust and reproducible radiomic features.
\end{abstract}

Keywords: HCC; MRI; radiomics; manual segmentation; semi-automatic segmentation

\section{Introduction}

Hepatocellular carcinoma (HCC) is of the common type of malignant tumour of the liver and its incidence has increased in recent years. In Malaysia, HCC is one of the top five cancers with $4.4 \%$ new cases in 2018, and it ranks fourth in leading causes of cancer death [1]. HCC known to have a poor survival rate despite availability varieties of clinical treatments [2]. Medical imaging techniques such as computed tomography (CT) and magnetic resonance imaging (MRI) are vital procedures in oncological treatment. Both CT scans and MRI have higher spatial resolution that has advantage in the early detection of tissues' abnormality in patients [2]. Imaging is extensively used in detection, diagnosis, and staging of cancer. Several studies have been conducted to improve the tumour diagnosis through proposed methods [3-6]. Although MRI is acknowledged for its advantages, the false-negative diagnosis from the examinations is inevitable, although several quantitative techniques have been introduced which are mostly pertaining to MR study. It is crucial 
for optimizing quantitative imaging feature extraction through computational approaches and developing decision support systems to accurately estimate the cancer stage. Hence, radiomics study are introduced and used to extract high number of quantitative image features [7].

Feature extraction is essential to obtain relevant information on input images and represents data in lower dimensionality space [8]. These features are extracted by using advanced mathematical algorithm which describes phenotypes of tumour that might not be able to perceive by naked eye. Previous studies have shown the importance of quantitative feature extraction in classifying the cancer stage of various types of cancer, including HCC [9-12]. In 2016, investigation of extraction of radiomics data was performed to predict the pathological response after chemotherapy [13]. Incorporations of radiomics and machine learning are essential for information extraction to aid in better prognosis. From shape, statistics, and texture features, 20 features were selected based on stability and variance.

Recently, uses of radiomics in cancer related field shows significant progress. Radiomics application is said to be one of the fundamental methods for machine learning development in the medical imaging field [14,15]. Extraction of radiomic features from various sources of medical images also overcomes the limitation of visual image interpretation [16]. Several literature reviews show data mining and predictive analysis have widened the scope of medical imaging [17-20]. This can facilitate prognostic models used in oncology. However, the poor reliability of radiomic features could affect research outcomes and become an obstacle for further use in models [21].

It is important to ensure the accuracy of quantitative features extracted from medical images. The process of tumour segmentation is one of the main obstacles for radiomics. Conventional manual segmentation is considered quite tedious and time consuming where quick and reliable segmentation techniques are required. Previous studies show that semiautomatic segmentation methods are preferred, as they are better alternatives to manual segmentation [22-24]. Comparative analysis of different types of image segmentation had been done for mammographic images in 2019. This study concludes flood filling algorithm has the best segmentation result compared to the watershed algorithm, the mean-shift algorithm, and the k-means algorithm [25]. Recently, a study was done for non-small cell lung cancer, radiomic features extracted for semi-automatic segmentation using a grow-cut algorithm implemented in 3D-Slicer, has high reproducibility and is more robust compared to manual segmentation [26]. Studies on CT and MR images prove that semi-automatic segmentation is robust, which indicates its ability to produce reliable and reproducible radiomic models $[27,28]$. Hence, in this study, we aim to evaluate the reproducibility and robustness of various segmentation techniques of MR HCC images based on the extract radiomic features.

\section{Materials and Methods}

In this study, we analysed robustness of features extracted from 30 patients (median age: $63 \mathrm{y} / \mathrm{o}$ ) diagnosed with HCC by two different methods of segmentations, manual and semi-automatic. We extracted 662 features using 3D Slicer (Boston, MA, USA) software for both segmentations [29]. Features extractions for manual and semi-automatic segmentations were compared and analyzed. Despite having small samples size $(n=30)$, numbers of sets for observations were examined to find the pattern on features extracted. Briefly, a total of 240 segmentations (120 manual segmentations and 120 semi-automatic segmentations) were analyzed in this study.

\subsection{Magnetic Resonance Imaging (MRI) of HCC Patients}

The image data was obtained from open archive for medical images of cancer, The Cancer Imaging Archive (TCIA) (Arkansas, USA) [30]. Ninety-seven patients verified with hepatocellular carcinoma, Stages I-IV, were retrieved. After reviewing the data images, patients who underwent CT scans were excluded, and only non-contrast enhanced 
T1-weighted $(\mathrm{T} 1 \mathrm{~W})$ images $(\mathrm{TR} / \mathrm{TE}=210 / 1.1 \mathrm{~ms})$ were chosen. A total of 30 patients diagnosed using MRI were included in this study. For the final study, 17 male (median age: 64 years; range 23-77 years) and 13 females (median age: 62 years; range $45-77$ years). Stages of HCC are divided into two groups, Stages I-II and Stages III-IV, respectively.

\subsection{Semi-Automatic Segmentation in 3D-Slicer}

For the semi-automatic segmentation, the flood fill algorithm from 3D Slicer software was applied on the subject MR images. Prior to the installation, the Segment Editor Extra Effects extension was installed through the extension's manager. After loading data images through the DICOM module, the observers identify the location of HCC. The nodes were added around the tumour region using a mouse cursor. Subsequently, the flood fill effects were activated, and ROI were segmented according to similar voxels intensity. To finalize the output, the segmented tumour was manually edited in the finalization phase as semi-automatic segmentation. Flood fill is an algorithm used to determine connection of an area in multi-dimensional array with the help of similarity of intensity voxels to the selected node determined by users. This algorithm is comparable with bucket tool in paint programs, which fill connected similar intensity voxels with different colours [25]. The algorithm was initiated with a start node by selecting the region of interest (ROI). Pixels connected four-directionally with the start node and pixels connected four-directionally to the former are considered in this effect. As the intensity voxels were decided, the algorithm identified the path of target node given and replaced it with different colours. Leakage prevention to other structures under this effect were manipulated using the neighbourhood size parameter.

\subsection{Feature Extraction}

All segmented image data were analysed and performed in the same software, 3D Slicer under the Radiomics module to extract imaging features. Features were extracted using the mathematical algorithm predicated on pixel intensities. From semi-automatic and manual segmentations, we defined 662 radiomic features for MR images that assist in quantifying tumour characteristics. The features were divided into four groups: (I) tumour intensity, (II) shape, (III) texture, and (IV) wavelet-features with examples of shape features shown in Table 1. The total features extracted from volume of interest for tumour intensity, shape and textural were 18, 14, and 54 features, respectively.

Table 1. Composition of 662 radiomic features extracted using 3D-Slicer.

\begin{tabular}{ccc}
\hline Features & Original $(\mathbf{n}=\mathbf{1})$ & Wavelet $(\mathbf{n}=\mathbf{8})$ \\
\hline Shape $(\mathrm{n}=14)$ & $14 \times 1$ & - \\
Texture $(\mathrm{n}=54)$ & $54 \times 1$ & $54 \times 8$ \\
Tumour Intensity $(\mathrm{n}=18)$ & $18 \times 1$ & $18 \times 8$ \\
\hline
\end{tabular}

Tumour intensity represents a first order statistic, which distinguishes a histogram of voxel intensity within the tumour region on MRI. Shape features are calculated and described with volume properties of the tumour. Shape features extracted using the pyRadiomics package are independent from gray-level intensities and can only be calculated on a non-derived image, which is the original image [31]. Thus, the wavelet filter is not applied to shape features as it will not be calculated on derived images. Textural features were determined by patterns or spatial distributions of voxel intensities derived from grey level dependence matrix (GLDM), grey level co-occurrence matrix (GLCM) and grey level run-length matrices (GLRLM) [32]. Features derived from co-occurrence and run-length matrices were computed by averaging all 13 symmetric directions in three dimensions [6].

Wavelet features are obtained by transforming domain representations of tumour intensity and textural features. These features were applied as either a high $(\mathrm{H})$ or low pass (L) filter in each of the three dimensions-X-axis, Y-axis, and Z-axis: wavelet-LHL, wavelet-LHH, wavelet-HLL, wavelet-LLH, wavelet-HLH, wavelet-HHH, wavelet-HHL, 
and wavelet-LLL [14]. Eight decomposed volumes of images were used on the intensity and textural features in the volume of interest, which resulted in a total of $576(8 \times 72)$ wavelet transforms features $[7,23]$. These filters help to identify the detail or sudden changes in intensity in the image [33]. Several fundamental formulas, such as entropy, contrast, uniformity, and correlation, are presented in the equation below:

$$
\text { Entropy }=-\sum_{i=1}^{N_{g}} p(i) \log _{2}(p(i)+\varepsilon)
$$

Entropy measures the average amount of information required to encode the image values, where $N_{g}=$ number of non-zero bins and $p(i)=$ normalized first order histogram.

$$
\text { Contrast }=\sum_{i=1}^{N_{g}} \sum_{j=1}^{N_{g}}(i-j)^{2} p(i, j)
$$

Contrast determines local intensity variation present in the image. A larger value correlates with greater disparity in intensity values among neighboring voxels.

$$
\text { Uniformity }=\sum_{i=1}^{N_{g}} p(i)^{2}
$$

Uniformity measures the sum of squares of each intensity value. Greater uniformity implies greater homogeneity. Correlation defines the linear dependency of grey level values to their respective voxels in GLCM. The value for correlation is between 0 (uncorrelated) and 1 (perfectly correlated). The equation of correlation is shown below:

$$
\text { Correlation }=\frac{\sum_{i=1}^{N_{g}} \sum_{j=1}^{N_{g}} p(i, j) i j-\mu_{x} \mu_{y}}{\sigma_{x}(i) \sigma_{y}(j)}
$$

where $\mu_{x}, \mu_{y}$ and $\sigma_{x}, \sigma_{y}$ are mean grey level intensity and standard deviation of $p_{x}$ and $p_{y}$, respectively.

\subsection{Statistical Analysis}

The intra-class correlation coefficient (ICC) is referred to as correlations within a class of data. It was calculated to quantify the reproducibility of features obtained. Figure 1 shows the flowcharts for reproducibility analysis in this study. ICC is a well-known statistical tool among researchers, ranging between 0 to 1 . There are three type of ICC models which can be chosen appropriately according to experimental situation. For this study, variance estimates were obtained to determine the ICC for inter-observer segmentations by using a two-way mixed effect model of analysis of variance (ANOVA) [25]. Equation below defines the ICC:

$$
\operatorname{ICC}(A, 1)=\frac{M S_{R}-M S_{E}}{M S_{R}+(k+1) M S_{g}+\frac{k}{n}\left(M S_{C}-M S_{E}\right)}
$$

One-way analysis of variance (ANOVA) was used to obtained the ICC values for intra-observer segmentation [15,16]. Equation below defines ICC $(C, 1)$ :

$$
\operatorname{ICC}(C, 1)=\frac{M S_{R}-M S_{W}}{M S_{R}+(k-1) M S_{W}}
$$

where $M S_{R}=$ mean square for rows, $M S_{W}=$ mean square for residual sources of variance, $M S_{E}=$ mean square error, $M S_{C}=$ mean square for columns, $k$ and $\mathrm{n}$ are number of observers involved and subjects. 


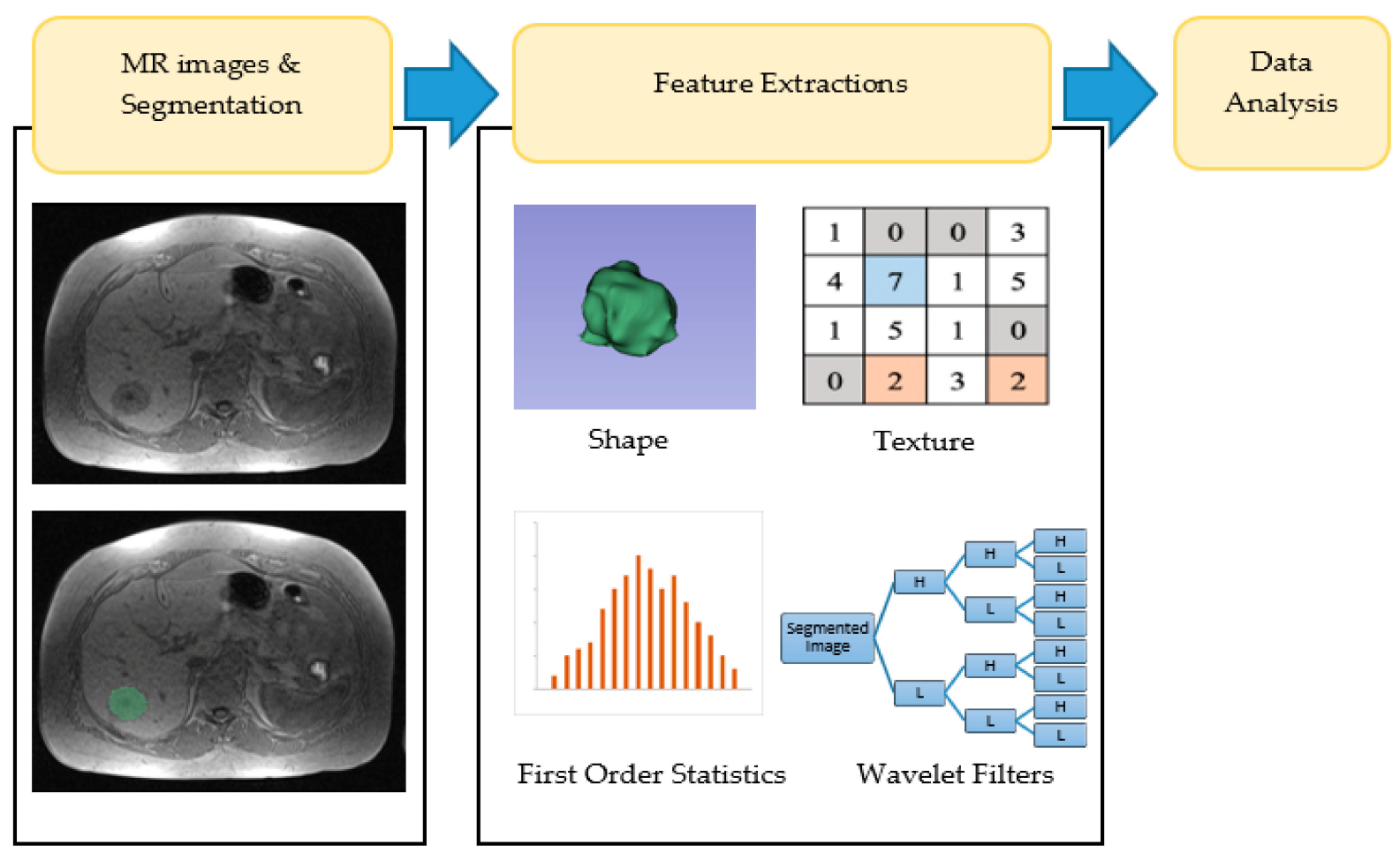

Figure 1. Schematic diagram of the analysis.

We evaluate intra-observers' reproducibility by letting one observer segmentized two months apart of 30 patients and this demonstrates variation of data measured from two different sets of segmentation. Furthermore, this also helps to assess multiple initializations of segmentation algorithm from the same observer. For inter-observers' reproducibility, the delineation was accomplished by several observers using the same methods of segmentation and the degree of agreement between different observers were analyzed. The difference of reproducibility for each segmentation were assessed using the Wilcoxon rank-sum test with $p$-value set at 0.05 . All data were expressed in mean \pm SD. All analyses of data were accomplished using Statistical Package for Social Sciences (SPSS, also known as IBM SPSS statistics) version 25 (SPSS Chicago, IL, USA).

\section{Results}

In order to investigate the reliability of radiomic features extracted from 3D Slicer segmentation on MR images, a total of 662 features under four groups, (I) tumour intensity, (II) shape, (III) texture, and (IV) wavelet-features, were assessed. Of 30 subjects, the size of tumours for Stages III-IV (average size $=8.9 \pm 3.4 \mathrm{~cm}$ ) are larger than Stages I-II (average size $=3.7 \pm 0.7 \mathrm{~cm}$ ). We extracted radiomic from volume of interest determined by two independent observers twice using 3D Slicer software semi-automatic segmentation and compared them to manual segmentation by four independent observers. The semi-automatic segmentations were divided into two sets, each having two segmentations. Figure 2 shows comparisons of intra-class correlation coefficient between semi-automatic and manual segmentation in terms of categorized features. We observed that features extracted from semi-automatic segmentation had significantly higher reproducibility (average ICC $=0.952 \pm 0.009, p<0.05)$ compared to features extracted from manual segmentation (average ICC $=0.897 \pm 0.011, p>0.05$ ). 


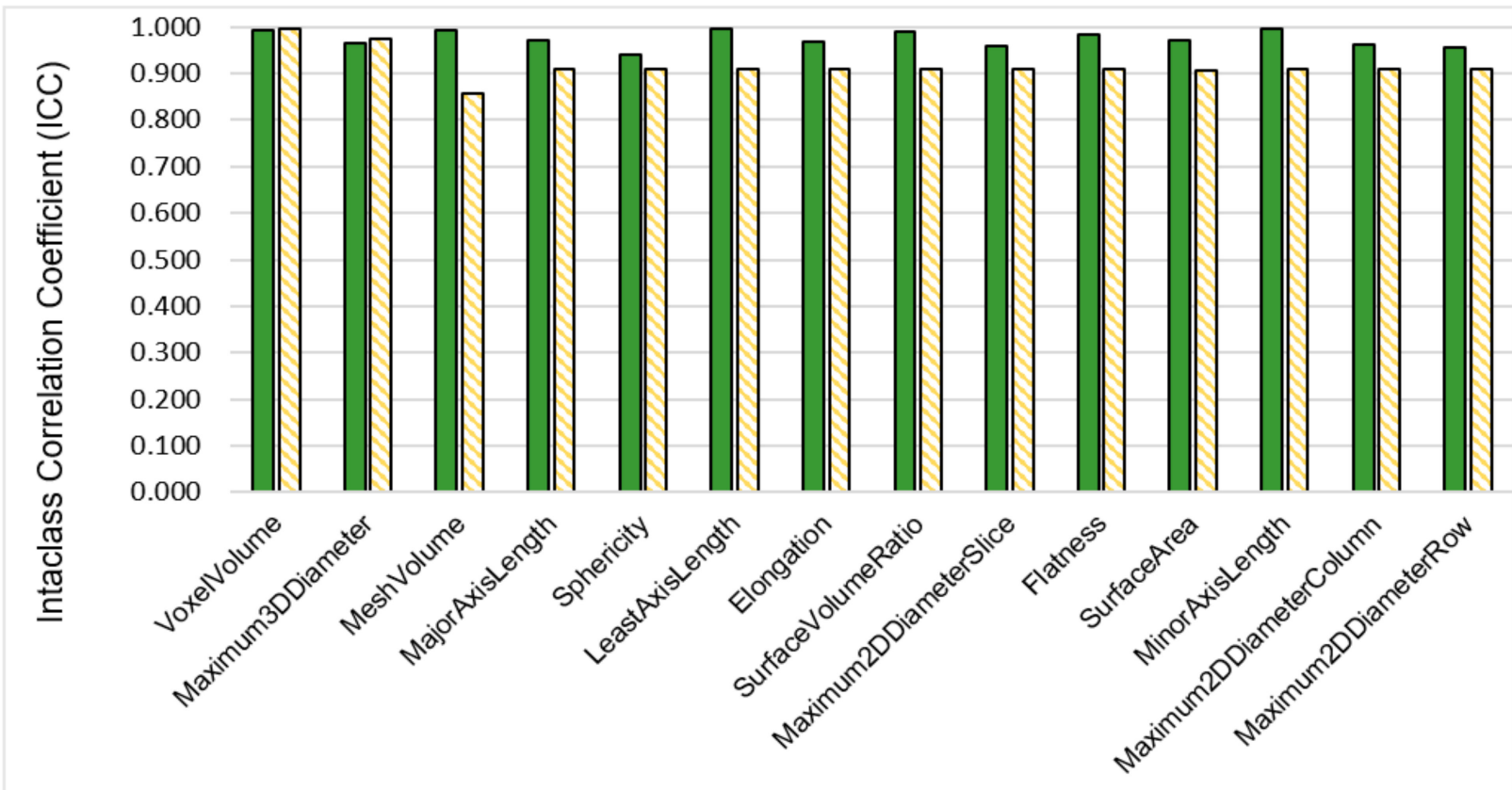

$\square$ Semi-auto Segmentation $\square$ Manual Segmentation

(a)

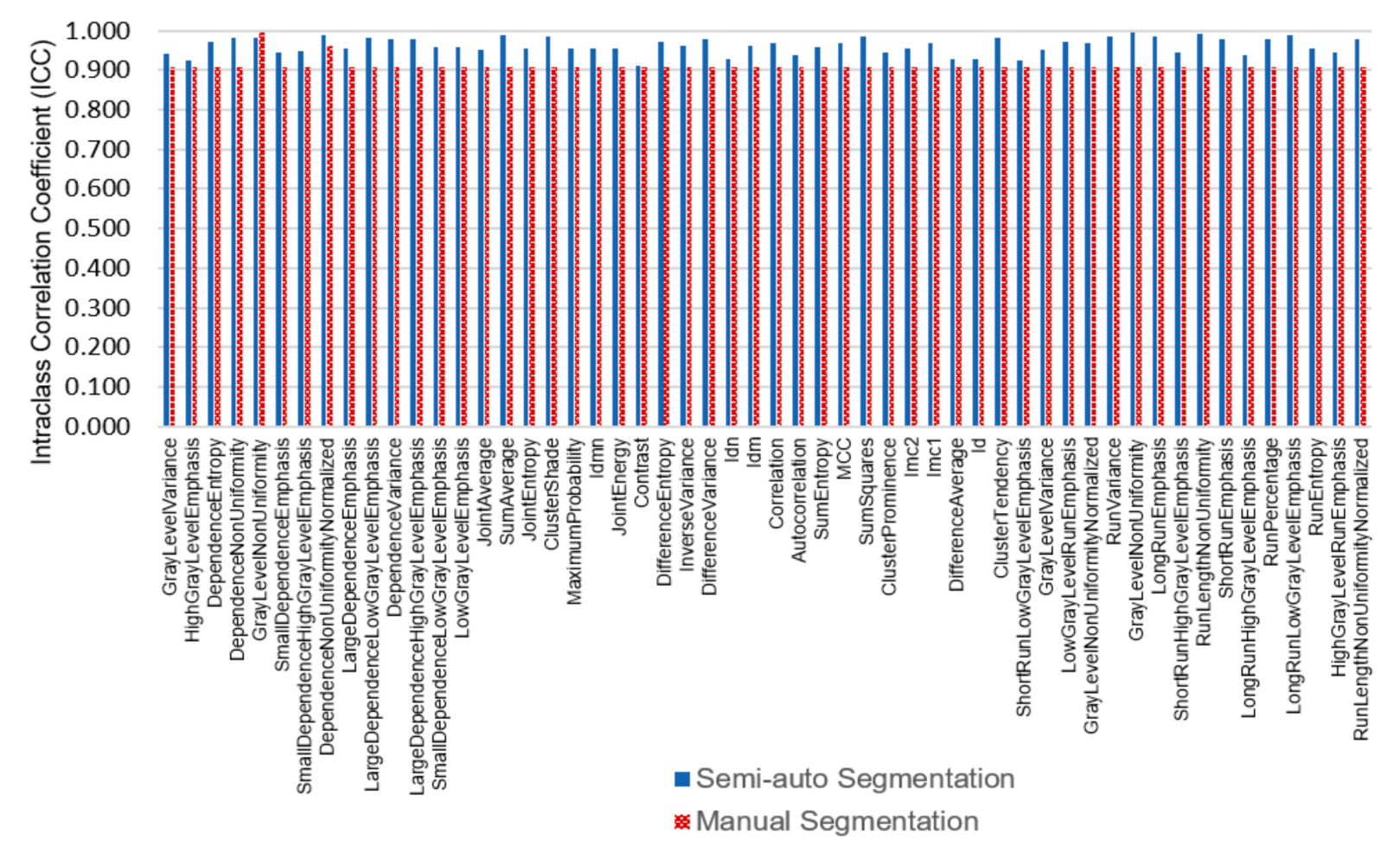

(b)

Figure 2. Cont. 


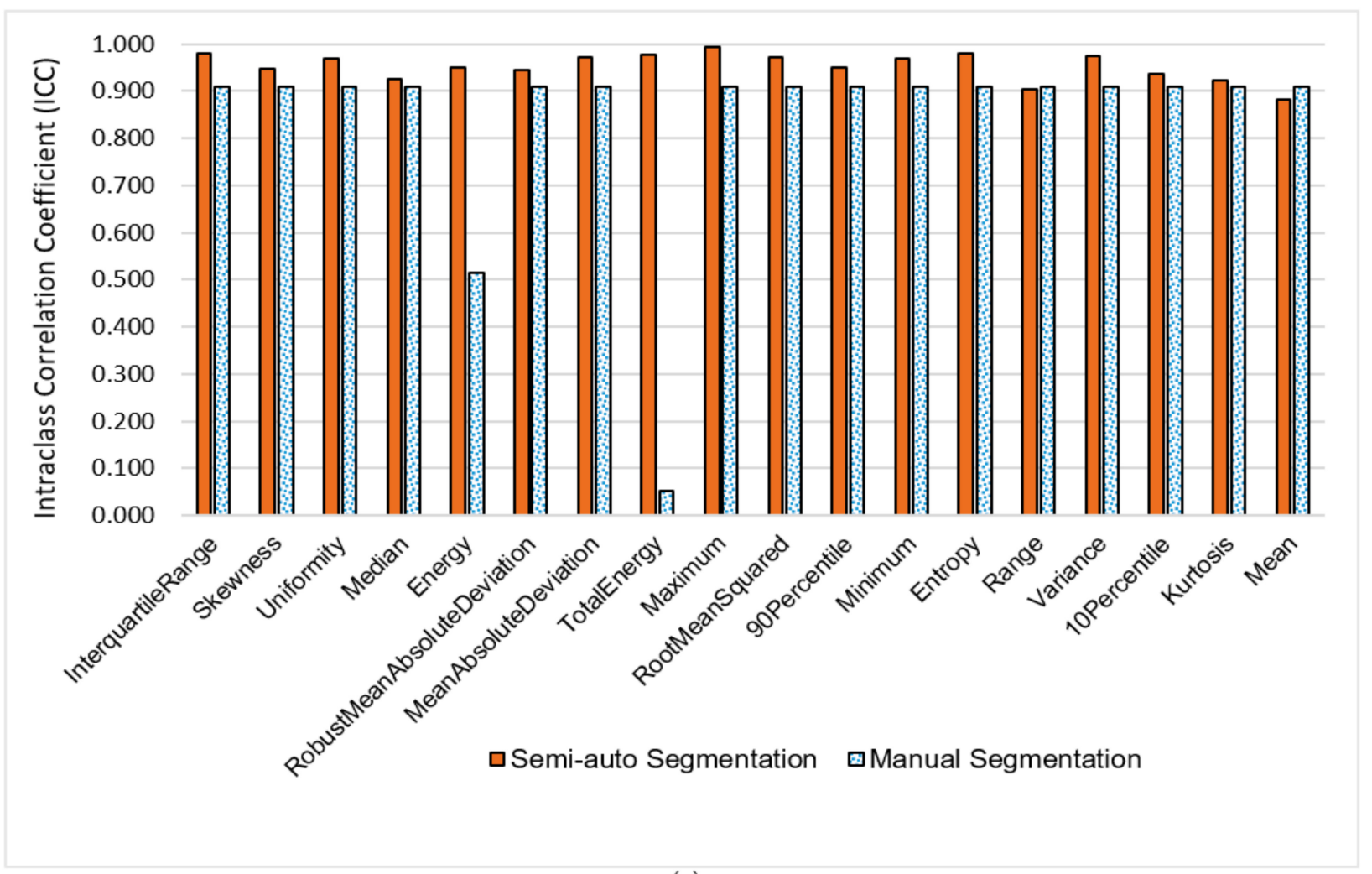

(c)

Figure 2. Comparisons of intra-class correlation coefficient (ICC) values in (a) shape based features, (b) textural features, and (c) first order statistics features.

There are 576 features of wavelet features, we separate the features into their respective feature types such as GLDM, GLCM, GLRLM and first order statistics for both semiautomatic and manual segmentations to ease the analysis process. There are 8 wavelet transforms in total. Figure 3 shows comparisons for ICC values of GLDM and first order features from both techniques. We observed that ICC values for intensity and texture features applied with wavelet transforms were high for semi-automatic segmentation compared to manual segmentation. Most ICC values for manual segmentations that undergoes wavelet transforms seen to have lower value which at lower level of the graphs. The ICC values of GLDM, GLCM, GLRLM, and intensity statistics for semi-automatic segmentation are $0.935 \pm 0.008,0.921 \pm 0.013,0.943 \pm 0.011$, and $0.948 \pm 0.008$, respectively. In contrary, ICC values for manual segmentations shows fluctuation in all four parameters (GLDM, GLCM, GLRLM, and intensity statistics) with average ICC equals to $0.796 \pm 0.032$, $0.848 \pm 0.021,0.941 \pm 0.012$, and $0.578 \pm 0.037$. Of the 662 features, 354 features $(53 \%)$ showed higher ICC values for semi-automatic segmentation compared to manual one. Tables $2-4$ show the reproducibility of ICC for both segmentations in regards with shape features and wavelet features. 


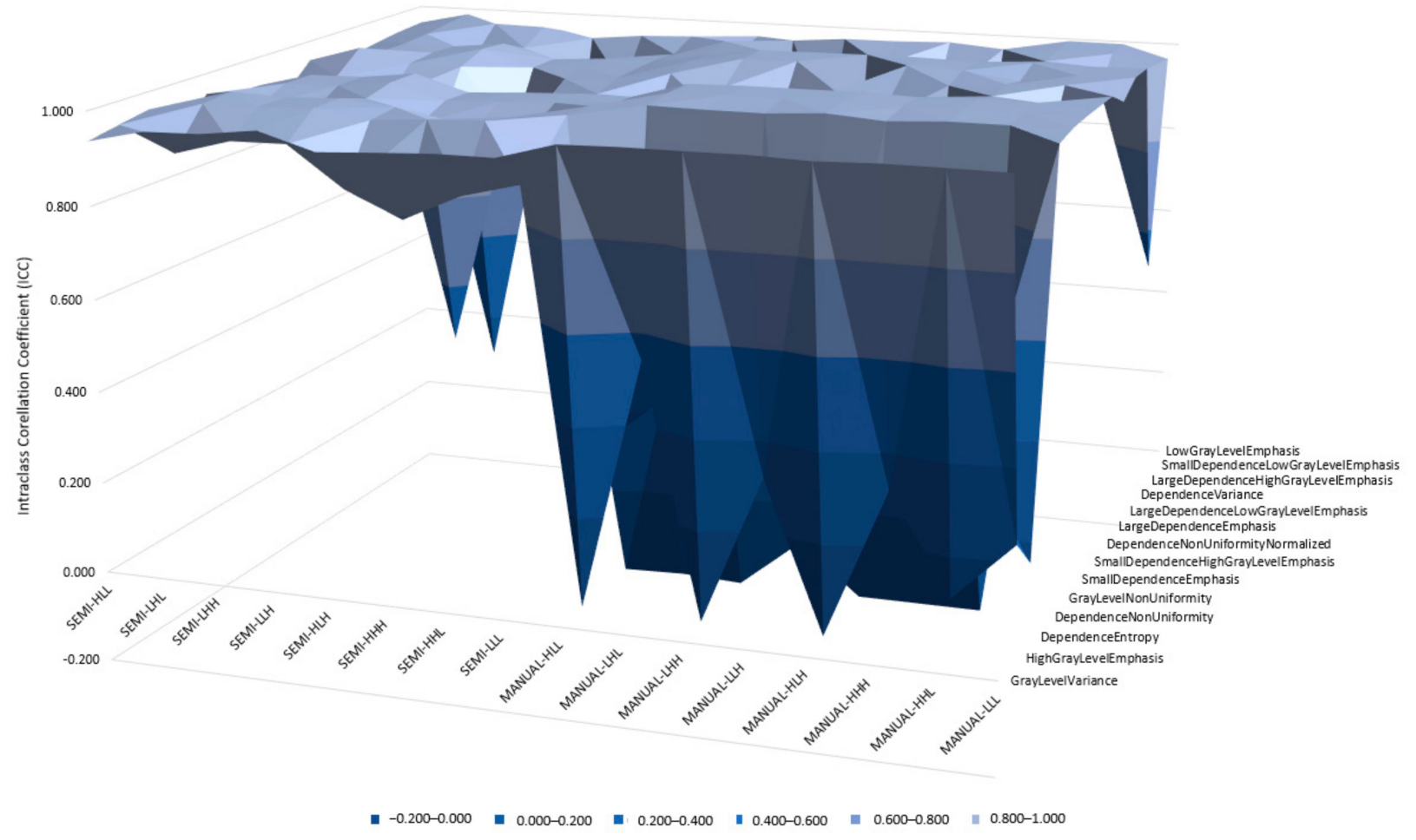

(a)

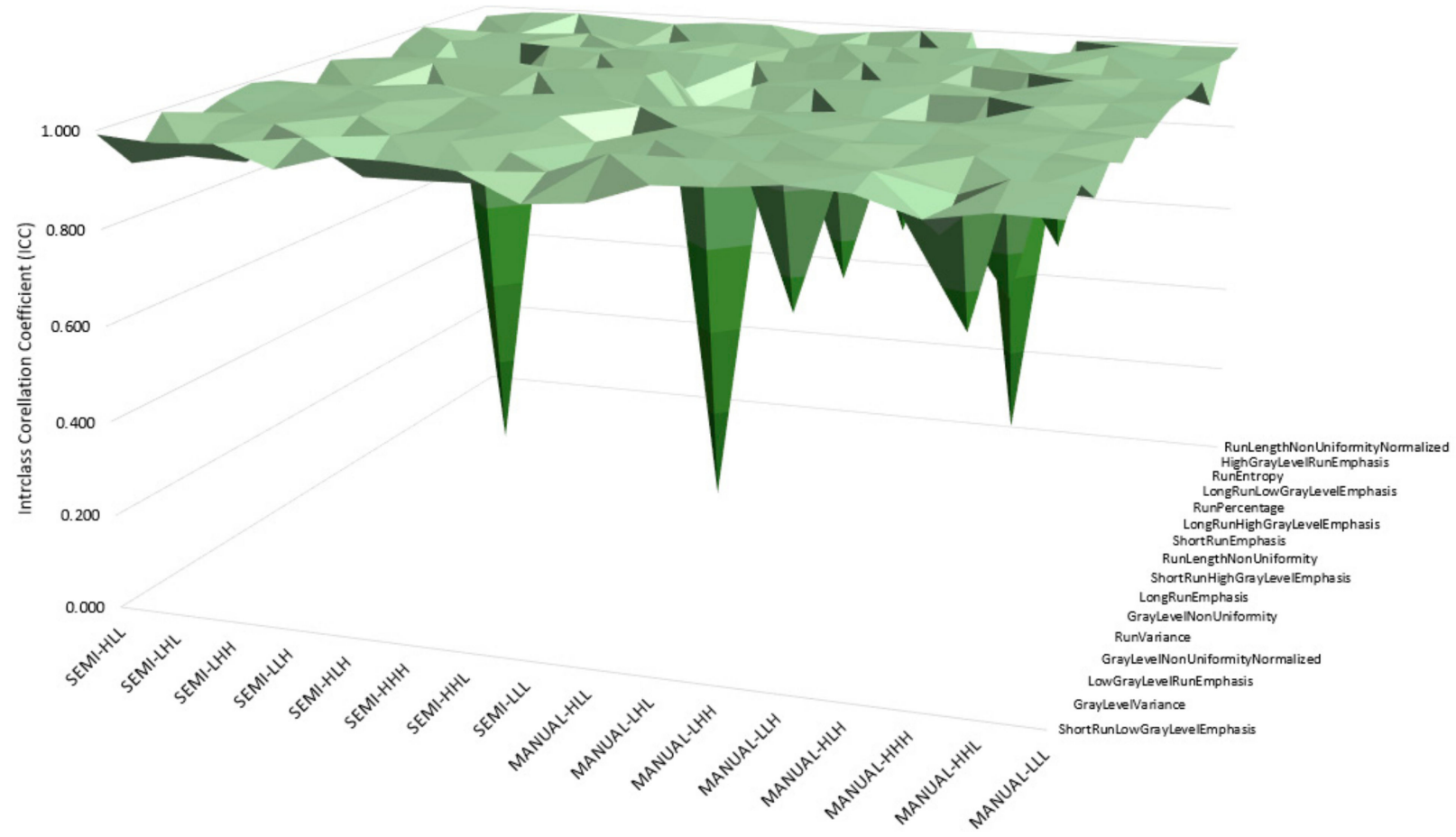

- $0.000-0.200=0.200-0.400=0.400-0.600=0.600-0.800=0.800-1.000$

(b)

Figure 3. Comparisons of wavelet features for semi-automatic and manual segmentation in; (a) Gray-level dependence matrix (GLDM) features and, (b) first order statistics features. 
Table 2. Intra-class correlation coefficient (ICC) of semi-automatic and manual segmentation for shape and grey level run length matrix (GLRLM) features.

\begin{tabular}{|c|c|c|c|}
\hline Features & Original & Semi-Automatic & Manual \\
\hline \multirow{14}{*}{ Shape } & Voxel Volume * & 0.994 & 0.996 \\
\hline & Maximum 3D-Diameter & 0.964 & 0.973 \\
\hline & Mesh Volume* & 0.994 & 0.856 \\
\hline & Major Axis Length & 0.972 & 0.909 \\
\hline & Sphericity & 0.940 & 0.909 \\
\hline & Least Axis Length * & 0.997 & 0.909 \\
\hline & Elongation & 0.968 & 0.909 \\
\hline & Surface Volume Ratio * & 0.990 & 0.909 \\
\hline & Maximum 2D-Diameter Slice & 0.960 & 0.909 \\
\hline & Flatness & 0.983 & 0.909 \\
\hline & Surface Area & 0.971 & 0.908 \\
\hline & Minor Axis Length * & 0.996 & 0.909 \\
\hline & Maximum 2D-Diameter Column & 0.961 & 0.909 \\
\hline & Maximum 2D-Diameter Row & 0.955 & 0.909 \\
\hline \multirow{16}{*}{$\begin{array}{l}\text { Gray-level run-length matrix } \\
\text { (GLRLM) }\end{array}$} & Short Run Low Gray-Level Emphasis & 0.925 & 0.909 \\
\hline & Gray-Level Variance & 0.951 & 0.909 \\
\hline & Low Gray-Level Run Emphasis * & 0.972 & 0.909 \\
\hline & Gray-Level Non-Uniformity Normalized & 0.970 & 0.909 \\
\hline & Run Variance * & 0.987 & 0.909 \\
\hline & Gray-Level Non-Uniformity * & 0.996 & 0.909 \\
\hline & Long-Run Emphasis & 0.985 & 0.909 \\
\hline & Short Run High Gray-Level Emphasis & 0.946 & 0.909 \\
\hline & Run-Length Non-Uniformity * & 0.991 & 0.909 \\
\hline & Short Run Emphasis & 0.980 & 0.909 \\
\hline & Long Run High Gray-Level Emphasis & 0.937 & 0.906 \\
\hline & Run Percentage $*$ & 0.978 & 0.909 \\
\hline & Long Run Low Gray-Level Emphasis * & 0.989 & 0.907 \\
\hline & Run Entropy & 0.954 & 0.909 \\
\hline & High Gray-Level Run-Emphasis & 0.946 & 0.909 \\
\hline & Run-Length Non-Uniformity Normalized * & 0.978 & 0.909 \\
\hline \multirow{18}{*}{$\begin{array}{c}\text { Tumour Intensity (First Order } \\
\text { Statistics) }\end{array}$} & Interquartile Range * & 0.980 & 0.909 \\
\hline & Skewness & 0.947 & 0.909 \\
\hline & Uniformity & 0.968 & 0.909 \\
\hline & Median & 0.925 & 0.909 \\
\hline & Energy * & 0.951 & 0.514 \\
\hline & Robust Mean Absolute Deviation & 0.945 & 0.909 \\
\hline & Mean Absolute Deviation & 0.971 & 0.909 \\
\hline & Total Energy * & 0.978 & 0.053 \\
\hline & Maximum * & 0.994 & 0.909 \\
\hline & Root Mean Squared & 0.972 & 0.909 \\
\hline & 90-Percentile & 0.949 & 0.909 \\
\hline & Minimum & 0.968 & 0.909 \\
\hline & Entropy & 0.980 & 0.909 \\
\hline & Range & 0.905 & 0.909 \\
\hline & Variance & 0.975 & 0.909 \\
\hline & 10-Percentile & 0.937 & 0.909 \\
\hline & Kurtosis & 0.922 & 0.909 \\
\hline & Mean & 0.881 & 0.909 \\
\hline
\end{tabular}

* Statistically significant $(p<0.05)$. 
Table 3. Wavelet features for semi-automatic segmentation.

\begin{tabular}{|c|c|c|c|c|c|c|c|c|c|}
\hline \multirow{2}{*}{ Features } & \multirow{2}{*}{ Wavelet } & \multicolumn{8}{|c|}{ Semi-Auto Segmentation } \\
\hline & & 1.HLL & 2.LHL & 3.LHH & 4.LLH & 5.HLH & 6.HHH & 7.HHL & 8.LLL \\
\hline \multirow{16}{*}{$\begin{array}{c}\text { Gray-level } \\
\text { run-length matrix } \\
\text { (GLRLM) }\end{array}$} & $\begin{array}{c}\text { Short Run Low Gray-Level } \\
\text { Emphasis }\end{array}$ & 0.992 & 0.983 & 0.989 & 0.947 & 0.981 & 0.979 & 0.970 & 0.915 \\
\hline & Gray-Level Variance & 0.911 & 0.934 & 0.930 & 0.988 & 0.917 & 0.918 & 0.921 & 0.953 \\
\hline & $\begin{array}{c}\text { Low Gray-Level Run } \\
\text { Emphasis }\end{array}$ & 0.995 & 0.979 & 0.986 & 0.949 & 0.978 & 0.974 & 0.961 & 0.915 \\
\hline & $\begin{array}{c}\text { Gray-Level Non-Uniformity } \\
\text { Normalized }\end{array}$ & 0.960 & 0.980 & 0.986 & 0.990 & 0.968 & 0.979 & 0.982 & 0.967 \\
\hline & Run Variance & 0.984 & 0.983 & 0.988 & 0.997 & 0.978 & 0.990 & 0.990 & 0.863 \\
\hline & Gray-Level Non Uniformity & 0.996 & 0.997 & 0.996 & 0.996 & 0.995 & 0.995 & 0.996 & 0.997 \\
\hline & Long Run Emphasis & 0.982 & 0.979 & 0.985 & 0.996 & 0.975 & 0.989 & 0.990 & 0.912 \\
\hline & $\begin{array}{c}\text { Short Run High Gray-Level } \\
\text { Emphasis }\end{array}$ & 0.919 & 0.946 & 0.836 & 0.899 & 0.821 & 0.920 & 0.926 & 0.935 \\
\hline & $\begin{array}{l}\text { Run-Length } \\
\text { Non-Uniformity }\end{array}$ & 0.990 & 0.995 & 0.996 & 0.994 & 0.992 & 0.996 & 0.997 & 0.883 \\
\hline & Short Run Emphasis & 0.975 & 0.986 & 0.990 & 0.989 & 0.977 & 0.988 & 0.989 & 0.000 \\
\hline & $\begin{array}{c}\text { Long Run High Gray-Level } \\
\text { Emphasis }\end{array}$ & 0.925 & 0.947 & 0.834 & 0.872 & 0.821 & 0.922 & 0.927 & 0.768 \\
\hline & Run Percentage & 0.974 & 0.989 & 0.002 & 0.988 & 0.978 & 0.989 & 0.990 & 0.980 \\
\hline & $\begin{array}{c}\text { Long Run Low Gray-Level } \\
\text { Emphasis }\end{array}$ & 0.998 & 0.971 & 0.971 & 0.936 & 0.971 & 0.966 & 0.943 & 0.908 \\
\hline & Run Entropy & 0.951 & 0.973 & 0.976 & 0.983 & 0.958 & 0.977 & 0.980 & 0.971 \\
\hline & $\begin{array}{c}\text { High Gray-Level Run } \\
\text { Emphasis }\end{array}$ & 0.920 & 0.947 & 0.836 & 0.896 & 0.820 & 0.921 & 0.927 & 0.935 \\
\hline & $\begin{array}{l}\text { Run-Length Non-Uniformity } \\
\text { Normalized }\end{array}$ & 0.974 & 0.990 & 0.993 & 0.987 & 0.978 & 0.988 & 0.989 & 0.971 \\
\hline \multirow{18}{*}{ First Order Statistics } & Interquartile Range & 0.989 & 0.988 & -0.001 & 0.991 & 0.975 & 0.926 & 0.990 & 0.989 \\
\hline & Skewness & 0.945 & 0.968 & 0.966 & 0.986 & 0.945 & 0.950 & 0.956 & 0.935 \\
\hline & Uniformity & 0.967 & 0.987 & 0.988 & 0.984 & 0.967 & 0.976 & 0.983 & 0.951 \\
\hline & Median & 0.903 & 0.936 & 0.665 & 0.977 & 0.916 & 0.917 & 0.921 & 0.923 \\
\hline & Energy & 0.912 & 0.934 & 0.929 & 0.988 & 0.916 & 0.917 & 0.921 & 0.953 \\
\hline & $\begin{array}{c}\text { Robust Mean Absolute } \\
\text { Deviation }\end{array}$ & 0.919 & 0.946 & 0.836 & 0.869 & 0.820 & 0.921 & 0.927 & 0.935 \\
\hline & Mean Absolute Deviation & 0.949 & 0.961 & 0.970 & 0.981 & 0.945 & 0.974 & 0.973 & 0.973 \\
\hline & Total Energy & 0.994 & 0.993 & 0.996 & 0.993 & 0.994 & 0.995 & 0.997 & 0.996 \\
\hline & Maximum & 0.994 & 0.994 & 0.994 & 0.994 & 0.994 & 0.994 & 0.994 & 0.994 \\
\hline & Root Mean Squared & 0.976 & 0.986 & 0.983 & 0.984 & 0.978 & 0.969 & 0.976 & 0.974 \\
\hline & 90-Percentile & 0.910 & 0.923 & 0.833 & 0.952 & 0.828 & 0.906 & 0.912 & 0.940 \\
\hline & Minimum & 0.976 & 0.983 & 0.970 & 0.982 & 0.975 & 0.933 & 0.963 & 0.965 \\
\hline & Entropy & 0.977 & 0.987 & 0.992 & 0.991 & 0.978 & 0.991 & 0.991 & 0.933 \\
\hline & Range & 0.999 & 0.976 & 0.977 & 0.965 & 0.970 & 0.965 & 0.942 & 0.845 \\
\hline & Variance & 0.989 & 0.996 & 0.987 & 0.986 & 0.993 & 0.953 & 0.975 & 0.867 \\
\hline & 10-Percentile & 0.954 & 0.909 & 0.858 & 0.770 & 0.987 & 0.898 & 0.913 & 0.949 \\
\hline & Kurtosis & 0.971 & 0.964 & 0.956 & 0.963 & 0.994 & 0.988 & 0.991 & 0.946 \\
\hline & Mean & 0.995 & 0.980 & 0.986 & 0.954 & 0.997 & 0.974 & 0.961 & 0.922 \\
\hline
\end{tabular}


Table 4. Wavelet features for manual segmentation.

\begin{tabular}{|c|c|c|c|c|c|c|c|c|c|}
\hline \multirow{2}{*}{ Features } & \multirow{2}{*}{ Wavelet } & \multicolumn{8}{|c|}{ Manual Segmentation } \\
\hline & & 1.HLL & 2.LHL & 3.LHH & 4.LLH & 5.HLH & 6.HHH & 7.HHL & 8.LLL \\
\hline \multirow{16}{*}{$\begin{array}{c}\text { Gray-level } \\
\text { run-length matrix } \\
(\text { GLRLM) }\end{array}$} & $\begin{array}{l}\text { Short Run Low Gray-Level } \\
\text { Emphasis }\end{array}$ & 0.926 & 0.969 & 0.974 & 0.987 & 0.980 & 0.942 & 0.963 & 0.960 \\
\hline & Gray-Level Variance & 0.998 & 0.999 & 0.999 & 0.999 & 0.998 & 0.887 & 1.000 & 0.998 \\
\hline & $\begin{array}{c}\text { Low Gray-Level Run } \\
\text { Emphasis }\end{array}$ & 0.924 & 0.965 & 0.972 & 0.987 & 0.979 & 0.938 & 0.957 & 0.950 \\
\hline & $\begin{array}{c}\text { Gray-Level Non-Uniformity } \\
\text { Normalized }\end{array}$ & 0.980 & 0.991 & 0.993 & 0.997 & 0.983 & 0.989 & 0.986 & 0.993 \\
\hline & Run Variance & 0.984 & 0.976 & 0.989 & 0.978 & 0.980 & 0.987 & 0.981 & 0.968 \\
\hline & Gray-Level Non Uniformity & 0.996 & 0.996 & 0.996 & 0.997 & 0.996 & 0.996 & 0.996 & 0.998 \\
\hline & Long Run Emphasis & 0.985 & 0.979 & 0.988 & 0.979 & 0.979 & 0.987 & 0.981 & 0.972 \\
\hline & $\begin{array}{c}\text { Short Run High Gray-Level } \\
\text { Emphasis }\end{array}$ & 0.872 & 0.516 & 0.927 & 0.716 & 0.506 & 0.999 & 0.980 & 0.984 \\
\hline & $\begin{array}{c}\text { Run-Length } \\
\text { Non-Uniformity }\end{array}$ & 0.990 & 0.991 & 0.995 & 0.991 & 0.993 & 0.996 & 0.994 & 0.991 \\
\hline & Short Run Emphasis & 0.983 & 0.990 & 0.994 & 0.996 & 0.984 & 0.990 & 0.986 & 0.989 \\
\hline & $\begin{array}{c}\text { Long Run High Gray-Level } \\
\text { Emphasis }\end{array}$ & 0.869 & 0.507 & 0.925 & 0.675 & 0.490 & 0.996 & 0.979 & 0.981 \\
\hline & Run Percentage & 0.982 & 0.992 & 0.995 & 0.996 & 0.986 & 0.991 & 0.988 & 0.988 \\
\hline & $\begin{array}{c}\text { Long Run Low Gray-Level } \\
\text { Emphasis }\end{array}$ & 0.915 & 0.939 & 0.962 & 0.955 & 0.969 & 0.938 & 0.945 & 0.914 \\
\hline & Run Entropy & 0.992 & 0.989 & 0.993 & 0.999 & 0.992 & 0.996 & 0.995 & 0.994 \\
\hline & $\begin{array}{c}\text { High Gray-Level Run } \\
\text { Emphasis }\end{array}$ & 0.872 & 0.515 & 0.927 & 0.708 & 0.504 & 0.999 & 0.980 & 0.984 \\
\hline & $\begin{array}{l}\text { Run-Length Non-Uniformity } \\
\text { Normalized }\end{array}$ & 0.983 & 0.993 & 0.995 & 0.001 & 0.987 & 0.992 & 0.989 & 0.991 \\
\hline \multirow{18}{*}{ First Order Statistics } & Interquartile Range & -0.005 & 0.021 & 0.087 & 0.033 & 0.010 & 0.026 & -0.007 & -0.008 \\
\hline & Skewness & 0.994 & 0.998 & 0.998 & 0.999 & 0.995 & 0.999 & 0.999 & 0.999 \\
\hline & Uniformity & 0.972 & 0.985 & 0.988 & 0.992 & 0.982 & 0.984 & 0.984 & 0.957 \\
\hline & Median & 0.998 & 0.999 & 0.999 & 0.999 & 0.999 & 1.000 & 1.000 & 0.997 \\
\hline & Energy & 0.000 & 0.000 & 0.000 & 0.000 & 0.000 & 0.000 & 0.000 & 0.000 \\
\hline & $\begin{array}{c}\text { Robust Mean Absolute } \\
\text { Deviation }\end{array}$ & 0.872 & 0.515 & 0.927 & 0.708 & 0.504 & 0.999 & 0.980 & 0.984 \\
\hline & Mean Absolute Deviation & 0.096 & 0.946 & 0.958 & 0.918 & 0.867 & 0.971 & 0.925 & 0.057 \\
\hline & Total Energy & 0.000 & 0.001 & 0.030 & 0.000 & 0.002 & 0.051 & 0.002 & 0.000 \\
\hline & Maximum & 0.996 & 0.996 & 0.996 & 0.997 & 0.996 & 0.996 & 0.996 & 0.997 \\
\hline & Root Mean Squared & -0.018 & 0.034 & 0.315 & 0.010 & -0.007 & 0.242 & 0.031 & -0.007 \\
\hline & 90-Percentile & 0.873 & 0.517 & 0.928 & 0.853 & 0.504 & 0.999 & 0.980 & 0.990 \\
\hline & Minimum & 0.001 & 0.006 & 0.007 & 0.013 & 0.000 & 0.004 & -0.001 & -0.025 \\
\hline & Entropy & 0.977 & 0.972 & 0.976 & 0.972 & 0.970 & 0.964 & 0.965 & 0.949 \\
\hline & Range & 0.548 & 0.953 & 0.979 & 0.787 & 0.965 & 0.927 & 0.939 & 0.183 \\
\hline & Variance & 0.102 & 0.912 & 0.981 & 0.837 & 0.560 & 0.974 & 0.630 & 0.065 \\
\hline & 10-Percentile & 0.888 & 0.718 & 0.890 & 0.600 & 0.587 & 0.964 & 0.969 & 0.921 \\
\hline & Kurtosis & -0.005 & -0.006 & -0.007 & -0.002 & -0.006 & -0.005 & -0.010 & 0.000 \\
\hline & Mean & 0.918 & 0.961 & 0.981 & 0.423 & 0.978 & 0.930 & 0.957 & 0.002 \\
\hline
\end{tabular}

Features that had been extracted are classified into three groups, high reproducibility (ICC $\geq 0.8)$, medium reproducibility $(0.8>$ ICC $\geq 0.5)$, and low reproducibility $($ ICC $<0.5)$, based on their ICC values. Out of 662 features, manual segmentation had $78.1 \%$ high, $6.5 \%$ medium, and $15.4 \%$ low reproducibility. However, semi-automatic segmentation, which is the flood filling effect, had $96.7 \%$ features in high reproducibility, $1.8 \%$ medium reproducibility, and $1.5 \%$ low reproducibility. Table 5 summarizes features extracted from both segmentations in reproducibility groups. Hence, reproducibility of the extracted features was higher for semi-automatic segmentations. 
Table 5. Features extracted in different reproducibility groups.

\begin{tabular}{ccc}
\hline Reproducibility Groups & Semi-Automatic & Manual \\
\hline High $($ ICC $\geq 0.8)$ & $639(96.7 \%)$ & $517(78.1 \%)$ \\
Medium $(0.8>$ ICC $\geq 0.5)$ & $12(1.8 \%)$ & $43(6.5 \%)$ \\
Low $($ ICC $<0.5)$ & $11(1.5 \%)$ & $102(15.4 \%)$ \\
\hline
\end{tabular}

The robustness of each technique was evaluated by analysing the ICC of features extracted from inter- and intra-observers. Based on Figure 4, high ICC values for interobserver from semi-automatic segmentation were observed (ICC $=0.976 \pm 0.006$ and ICC $=0.978 \pm 0.003$, respectively). Table 6 tabulates the inter-observer reproducibility of shape features for one subject.

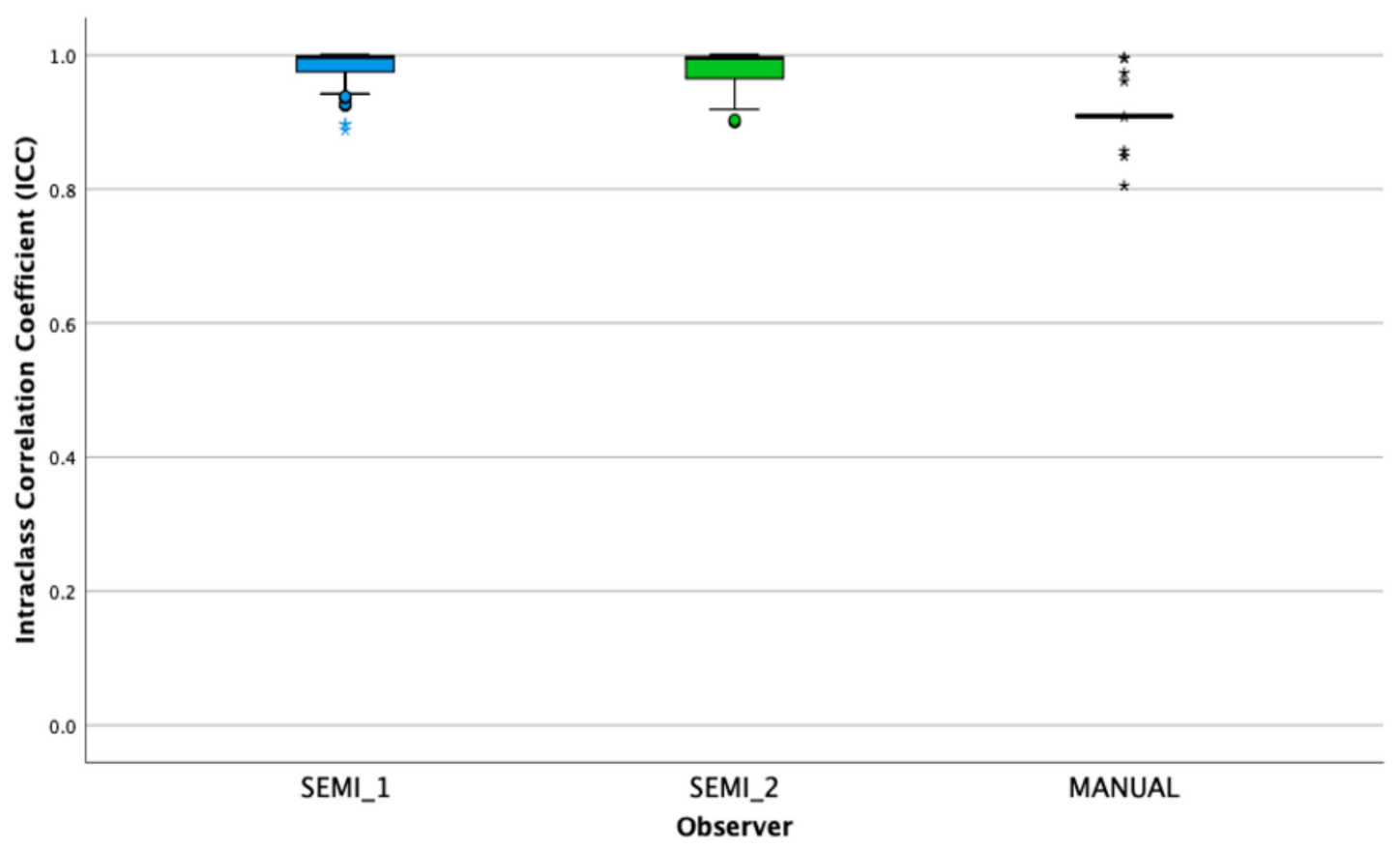

Figure 4. Boxplot of the ICC value represents by inter-observers' reproducibility on the radiomic features.

Table 6. ICC of radiomic features.

\begin{tabular}{cccc}
\hline Features & Semi_1 & Semi_2 & Manual \\
\hline Voxel Volume * & 0.984 & 0.996 & 0.996 \\
Maximum 3D-Diameter & 0.878 & 0.965 & 0.973 \\
Mesh Volume & 0.985 & 0.996 & 0.956 \\
Major Axis Length & 0.939 & 0.903 & 0.957 \\
Sphericity & 0.756 & 0.999 & 0.909 \\
Least Axis Length & 0.991 & 0.932 & 0.909 \\
Elongation * & 0.941 & 0.977 & 0.909 \\
Surface Volume Ratio & 0.962 & 0.944 & 0.909 \\
Maximum 2D-Diameter Slice & 0.924 & 0.974 & 0.989 \\
Flatness & 0.941 & 0.998 & 0.908 \\
Surface Area & 0.984 & 0.986 & 0.995 \\
\end{tabular}


Table 6. Cont.

\begin{tabular}{|c|c|c|c|c|}
\hline \multicolumn{2}{|r|}{ Features } & \multirow{2}{*}{$\begin{array}{c}\text { Semi_1 } \\
0.964\end{array}$} & \multirow{2}{*}{$\begin{array}{c}\text { Semi_2 } \\
0.927\end{array}$} & \multirow{2}{*}{$\begin{array}{c}\text { Manual } \\
0.909\end{array}$} \\
\hline \multirow{16}{*}{$\begin{array}{l}\text { Gray-level run-length } \\
\text { matrix (GLRLM) }\end{array}$} & $\begin{array}{c}\text { Short Run Low Gray-Level } \\
\text { Emphasis * }\end{array}$ & & & \\
\hline & Gray-Level Variance & 0.999 & 0.998 & 0.909 \\
\hline & Low Gray-Level Run Emphasis & 0.983 & 0.962 & 0.909 \\
\hline & $\begin{array}{c}\text { Gray-Level Non-Uniformity } \\
\text { Normalized }\end{array}$ & 0.997 & 0.998 & 0.909 \\
\hline & Run Variance & 0.993 & 0.999 & 0.909 \\
\hline & Gray-Level Non-Uniformity & 0.995 & 0.995 & 0.909 \\
\hline & Long-Run Emphasis & 0.992 & 0.999 & 0.909 \\
\hline & $\begin{array}{c}\text { Short Run High Gray-Level } \\
\text { Emphasis }\end{array}$ & 0.943 & 0.927 & 0.909 \\
\hline & Run-Length Non-Uniformity & 0.996 & 0.998 & 0.909 \\
\hline & Short Run Emphasis & 0.999 & 0.999 & 0.909 \\
\hline & $\begin{array}{l}\text { Long Run High Gray-Level } \\
\text { Emphasis * }\end{array}$ & 0.937 & 0.922 & 0.909 \\
\hline & Run Percentage & 0.999 & 0.999 & 0.909 \\
\hline & $\begin{array}{c}\text { Long Run Low Gray-Level } \\
\text { Emphasis }\end{array}$ & 0.986 & 0.998 & 0.909 \\
\hline & Run Entropy & 0.997 & 0.998 & 0.909 \\
\hline & High Gray-Level Run-Emphasis * & 0.943 & 0.927 & 0.909 \\
\hline & $\begin{array}{l}\text { Run-Length Non-Uniformity } \\
\text { Normalized }\end{array}$ & 0.999 & 0.999 & 0.909 \\
\hline & Interquartile Range & 0.98 & 0.989 & 0.909 \\
\hline & Skewness & 0.999 & 0.996 & 0.909 \\
\hline & Uniformity & 1 & 0.994 & 0.909 \\
\hline & Median & 1 & 0.993 & 0.909 \\
\hline & Energy & 0.999 & 0.998 & 0.805 \\
\hline & Robust Mean Absolute Deviation & 0.942 & 0.926 & 0.909 \\
\hline & Mean Absolute Deviation & 0.991 & 0.998 & 0.909 \\
\hline & Total Energy & 1 & 0.992 & 0.849 \\
\hline Tumour Intensity (First & Maximum & 0.999 & 0.992 & 0.909 \\
\hline Order Statistics) & Root Mean Squared & 0.997 & 0.995 & 0.909 \\
\hline & 90-Percentile * & 0.945 & 0.919 & 0.909 \\
\hline & Minimum & 0.995 & 0.987 & 0.909 \\
\hline & Entropy & 0.999 & 0.999 & 0.909 \\
\hline & Range & 0.998 & 0.998 & 0.909 \\
\hline & Variance & 0.999 & 0.997 & 0.909 \\
\hline & 10-Percentile & 0.944 & 0.928 & 0.909 \\
\hline & Kurtosis & 0.888 & 0.9 & 0.909 \\
\hline & Mean & 0.988 & 0.975 & 0.909 \\
\hline
\end{tabular}

* Statistically significant $(p<0.05)$.

\section{Discussion}

MR images were used in this study, as it produces high quality images compared to CT scans [34]. It is reported that resolution of input images influences the robustness of radiomic features as higher resolution images enhance visualization for segmentations [35]. In 2014, Chintan et al. assessed reproducibility of feature extracted using 3D-Slicer segmentation (GrowCut algorithm) which resulted in semi-automatic segmentation higher than manual delineation of CT images [7]. Therefore, additional features such as wavelet transform are implemented to study MR images using different types of algorithms. Despite increasing development in application of radiomic studies, robustness and reproducibility of radiomic features extracted from MR images are still one of the main challenges' oncologist encounters.

In this study, we investigate the reproducibility and robustness of radiomic features of HCC between two types of segmentations, manual and semi-automatic, using 3D-Slicer software. A total of 662 features were extracted from volume of interest and can be classified 
into four main groups (18 tumour intensity, 14 shape features, 54 textural features, and 576 wavelet features). Based on the results, semi-automatic segmentation has higher ICC values compared to manual segmentation. Semi-automatic segmentation, which we used for flood filling algorithm, is also more robust and significantly higher compared to manual segmentation. Flood filling algorithm was performed when the observer chose several points around the volume of interest, and the tumour region was digitized according to the uniform colour which results in accurate segmentation [30]. This algorithm is proven to be the best segmentation method by comparing their accuracy in selection of objects [25].

All four quantitative imaging features extracted from semi-automatic segmentation had higher reproducibility score $(p<0.05)$ compared to manual segmentation. For most tumour intensity feature, semi-automatic segmentation had higher reproducibility compared to manual segmentation. There were a few negative ICC values for wavelet features in both segmentations shown in Figure 3, which indicates that particular features vary than any features randomly chosen from the whole population [36]. Despite that, the reproducibility of wavelet features from semi-automatic segmentation is still higher compared to manual segmentation. Furthermore, semi-automatic segmentation was consistent with ICC values (97\% good reproducibility) compared to manual segmentation (78\% good reproducibility). In 2020, Wang et al. implied that the features' reliability increased using wavelet filters through their proposed deep learning tools compared to normal features [21].

We also analysed intra- and inter-observer reproducibility to evaluate the performance for both segmentations. Two sets from two different observers are shown in Figure 4 with one observer segments without formal clinical training. Despite using semi-automatic segmentation, experience of observers affects the segmentations of tumours as human interaction's influence the process of segmentation. this indicates the difference in radiomic features. However, semi-automatic segmentation demonstrates high ICC values for both intra- and inter-observers compared to manual segmentation. This suggests that features extracted from semi-automatic segmentation are more robust and reproducible. There are two limitations in this study. First, this study does not involve image descriptors for prognostic models due to the insufficient images of HCC patients from the accessible source. Secondly, this study only comprises of single MRI sequence. The contrast enhanced MRI and infiltrative HCC are not included in this study.

\section{Conclusions}

This study assesses the reproducibility of radiomic features between two techniques. Semi-automatic segmentation using the flood fill algorithm produces more reproducible features, and this indicates it could be a better alternative to the current manual segmentation. Thus, this algorithm could be further applied for prognostic and classification models with sufficient data taken from several centers. Note that the study was focused on robustness and reproducibility of features extracted from semi-automatic and manual segmentation. Hence, for adapting machine learning, the study should be in larger scales with more data so the application will be much accurate.

Author Contributions: Conceptualization, M.K.A.K.; formal analysis, N.S.M.H.; funding acquisition, M.K.A.K. and M.J.I.; investigation, M.K.A.K. and N.H.O.; methodology, N.S.M.H. and M.I.S.; resources, I.N.C.I.; software, I.N.C.I.; validation, M.K.A.K.; writing—original draft, N.S.M.H.; writingreview and editing, M.K.A.K. and M.J.I. All authors have read and agreed to the published version of the manuscript.

Funding: This work was supported by Ministry of Higher Education (MOHE) Malaysia under the Fundamental Research Grant Scheme (FRGS) with grant number FRGS/1/2020/STG07/UPM/02/3.

Institutional Review Board Statement: Not applicable.

Informed Consent Statement: Not applicable.

Acknowledgments: The authors would like to acknowledge Universiti Putra Malaysia and Ministry of Higher Education Malaysia (MOHE) for the financial support of this research. 
Conflicts of Interest: The authors declare no conflict of interest.

\section{References}

1. Azizah, A.M.; Hashimah, B.; Nirmal, K.; Siti Zubaidah, A.R.; Puteri, N.A.; Nabihah, A.; Sukumaran, R.; Balqis, B.; Nadia, S.M.R.; Sharifah, S.S.S.; et al. Malaysian National Cancer Registry Report; Ministry of Health: Putrajaya, Malaysia, 2017; pp. 1-116.

2. Kharrat, A.; Gasmi, K.; Ben Messaoud, M.; Benamrane, N.; Abid, M. A Hybrid Approach for Automatic Classification of Brain MRI Using Genetic Algorithm and Support Vector Machine An Hybrid Approach for Automatic Classification of Brain MRI Using Genetic Algorithm and Support Vector Machine. Leonardo J. Sci. 2010, 17, 71-82.

3. Ma, J.; Wang, Q.; Ren, Y.; Hu, H.; Zhao, J. Automatic lung nodule classification with radiomics approach. In Medical Imaging 2016: PACS and Imaging Informatics: Next Generation and Innovations; International Society for Optics and Photonics: California, CA, USA, 2016; Volume 9789, p. 978906. [CrossRef]

4. Vauthey, J.N.; Lauwers, G.Y.; Esnaola, N.F.; Do, K.A.; Belghiti, J.; Mirza, N.; Curley, S.A.; Ellis, L.M.; Regimbeau, J.M.; Rashid, A.; et al. Simplified staging for hepatocellular carcinoma. J. Clin. Oncol. 2002, 20, 1527-1536. [CrossRef] [PubMed]

5. Scrivener, M.; De Jong, E.E.C.; van Timmeren, J.E.; Pieters, T.; Geets, X. Radiomics applied to lung cancer: A review. Transl. Cancer Res. 2016, 5, 398-409. [CrossRef]

6. Xu, L.; Gao, Q.; Yousefi, N. Brain tumor diagnosis based on discrete wavelet transform, gray-level co-occurrence matrix, and optimal deep belief network. Simulation 2020, 96, 867-879. [CrossRef]

7. Parmar, C.; Velazquez, E.R.; Leijenaar, R.; Jermoumi, M.; Carvalho, S.; Mak, R.H.; Mitra, S.; Shankar, B.U.; Kikinis, R.; Haibe-Kains, B.; et al. Robust radiomics feature quantification using semiautomatic volumetric segmentation. PLoS ONE 2014, 9, e0102107. [CrossRef] [PubMed]

8. Kumar, G.; Bhatia, P.K. A detailed review of feature extraction in image processing systems. In Proceedings of the 2014 Fourth International Conference on Advanced Computing \& Communication Technologies, Rohtak, India, 8-9 February 2014; pp. 5-12. [CrossRef]

9. Chen, W.; Liu, B.; Peng, S.; Sun, J.; Qiao, X. Computer-Aided Grading of Gliomas Combining Automatic Segmentation and Radiomics. Int. J. Biomed. Imaging 2018, 2018, 2512037. [CrossRef] [PubMed]

10. Delzell, D.A.P.; Magnuson, S.; Peter, T.; Smith, M.; Smith, B.J. Machine Learning and Feature Selection Methods for Disease Classification with Application to Lung Cancer Screening Image Data. Front. Oncol. 2019, 9, 1-8. [CrossRef]

11. Oyama, A.; Hiraoka, Y.; Obayashi, I.; Saikawa, Y.; Furui, S.; Shiraishi, K.; Kumagai, S.; Hayashi, T.; Kotoku, J. Hepatic tumor classification using texture and topology analysis of non-contrast-enhanced three-dimensional T1-weighted MR images with a radiomics approach. Sci. Rep. 2019, 9, 2-11. [CrossRef]

12. Dutra, L.V.; Huber, R. Feature extraction and selection for ers-1/2 insar classification. Int. J. Remote Sens. 1999, $20,993-1016$. [CrossRef]

13. Coroller, T.P.; Agrawal, V.; Huynh, E.; Narayan, V.; Lee, S.W.; Mak, R.H.; Aerts, H.J.W.L. Radiomic-Based Pathological Response Prediction from Primary Tumors and Lymph Nodes in NSCLC. J. Thorac. Oncol. 2017, 12, 467-476. [CrossRef]

14. Çinarer, G.; Gürsel, B.; Haşim, A. Prediction of glioma grades using deep learning with wavelet radiomic features. Appl. Sci. 2020, 10, 6296. [CrossRef]

15. Izdihar, K.; Karim, M.K.A.; Aresli, N.N.; Radzi, S.F.M.; Sabarudin, A.; Yunus, M.M.; Rahman, M.A.; Shamsul, S. Detection of Novel Coronavirus from Chest X-Ray Radiograph Images via Automated Machine Learning and CAD4COVID. In Proceedings of the 2021 International Congress of Advanced Technology and Engineering (ICOTEN), Taiz, Yemen, 4-5 July 2021; pp. 1-4.

16. Haniff, N.S.M.; Karim, M.K.B.A.; Ali, N.S.; Rahman, M.A.A.; Osman, N.H.; Saripan, M.I. Magnetic Resonance Imaging Radiomics Analysis for Predicting Hepatocellular Carcinoma. In Proceedings of the 2021 International Congress of Advanced Technology and Engineering (ICOTEN), Taiz, Yemen, 4-5 July 2021; pp. 1-5.

17. Cao, X.H.; Stojkovic, I.; Obradovic, Z. A robust data scaling algorithm to improve classification accuracies in biomedical data. BMC Bioinf. 2016, 17, 359. [CrossRef]

18. El-Dahshan, E.A.S.; Mohsen, H.M.; Revett, K.; Salem, A.B.M. Computer-aided diagnosis of human brain tumor through MRI: A survey and a new algorithm. Expert Syst. Appl. 2014, 41, 5526-5545. [CrossRef]

19. Zhou, T.; Ruan, S.; Canu, S. A review: Deep learning for medical image segmentation using multi-modality fusion. Array 2019, 3-4, 100004. [CrossRef]

20. Simi, V.R.; Joseph, J. Segmentation of Glioblastoma Multiforme from MR Images-A comprehensive review. Egypt. J. Radiol. Nucl. Med. 2015, 46, 1105-1110. [CrossRef]

21. Wang, L.; Tan, J.; Ge, Y.; Tao, X.; Cui, Z.; Fei, Z.; Lu, J.; Zhang, H.; Pan, Z. Assessment of liver metastases radiomic feature reproducibility with deep-learning-based semi-automatic segmentation software. Acta Radiol. 2021, 62, 291-301. [CrossRef] [PubMed]

22. McGraw, K.O.; Wong, S.P. "Forming inferences about some intraclass correlations coefficients": Correction. Psychol. Methods 1996, 1, 390. [CrossRef]

23. Zhao, S.; Ren, W.; Zhuang, Y.; Wang, Z. The Influence of Different Segmentation Methods on the Extraction of Imaging Histological Features of Hepatocellular Carcinoma CT. J. Med. Syst. 2019, 43, 1-7. [CrossRef] [PubMed] 
24. Mat Radzi, S.F.; Abdul Karim, M.K.; Saripan, M.I.; Abd Rahman, M.A.; Osman, N.H.; Dalah, E.Z.; Mohd Noor, N. Impact of Image Contrast Enhancement on Stability of Radiomics Feature Quantification on a 2D Mammogram Radiograph. IEEE Access 2020, 8, 127720-127731. [CrossRef]

25. Podgornova, Y.A.; Sadykov, S.S. Comparative analysis of segmentation algorithms for the allocation of microcalcifications on mammograms. CEUR Workshop Proc. 2019, 2391, 122-127. [CrossRef]

26. Qiu, Q.; Duan, J.; Gong, G.; Lu, Y.; Li, D.; Lu, J.; Yin, Y. Reproducibility of radiomic features with GrowCut and GraphCut semiautomatic tumor segmentation in hepatocellular carcinoma. Transl. Cancer Res. 2017, 6, 940-948. [CrossRef]

27. Haarburger, C.; Müller-Franzes, G.; Weninger, L.; Kuhl, C.; Truhn, D.; Merhof, D. Radiomics feature reproducibility under inter-rater variability in segmentations of CT images. Sci. Rep. 2020, 10, 1-10. [CrossRef] [PubMed]

28. Lebre, M.A.; Vacavant, A.; Grand-Brochier, M.; Rositi, H.; Strand, R.; Rosier, H.; Abergel, A.; Chabrot, P.; Magnin, B. A robust multi-variability model based liver segmentation algorithm for CT-scan and MRI modalities. Comput. Med. Imaging Graph. 2019, 76, 101635. [CrossRef]

29. Fedorov, A.; Beichel, R.; Kalpathy-Cramer, J.; Finet, J.; Fillion-Robin, J.C.; Pujol, S.; Bauer, C.; Jennings, D.; Fennessy, F.; Sonka, M.; et al. 3D Slicer as an image computing platform for the Quantitative Imaging Network. Magn. Reson. Imaging. 2012, 30, 1323-1341. [CrossRef]

30. Clark, K.; Vendt, B.; Smith, K.; Freymann, J.; Kirby, J.; Koppel, P.; Moore, S.; Phillips, S.; Maffitt, D.; Pringle, M.; et al. The cancer imaging archive (TCIA): Maintaining and operating a public information repository. J. Digit. Imaging 2013, 26, 1045-1057. [CrossRef]

31. 3D Slicer. Slicer-Community 3D Slicer Documentation; 3D Slicer: Boston, MA, USA, 2019; pp. 2-48.

32. Zhou, J.; Lu, J.; Gao, C.; Zeng, J.; Zhou, C.; Lai, X.; Cai, W.; Xu, M. Predicting the response to neoadjuvant chemotherapy for breast cancer: Wavelet transforming radiomics in MRI. BMC Cancer 2020, 20, 100. [CrossRef]

33. Sudarshan, V.K.; Mookiah, M.R.K.; Acharya, U.R.; Chandran, V.; Molinari, F.; Fujita, H.; Ng, K.H. Application of wavelet techniques for cancer diagnosis using ultrasound images: A Review. Comput. Biol. Med. 2016, 69, 97-111. [CrossRef] [PubMed]

34. Kwong, R.Y.; Yucel, E.K. Computed Tomography Scan and Magnetic Resonance Imaging. Circulation 2003, 108, 1-3. [CrossRef] [PubMed]

35. Coenegrachts, K. Magnetic resonance imaging of the liver: New imaging strategies for evaluating focal liver lesions. World J. Radiol. 2009, 1, 72. [CrossRef]

36. Taylor, P.J. An Introduction to Intraclass Correlation that Resolves Some Common Confusions. Programs Sci. Technol. Values Crit. Creat. Thinking Public Policy 2009, 7656, 1-9. 